\title{
Research Paper \\ Relationship Between ZAP-70 Gene (rs104893674 "A/C") Polymorphism With Risk for Acute Myeloid Leukemia
}

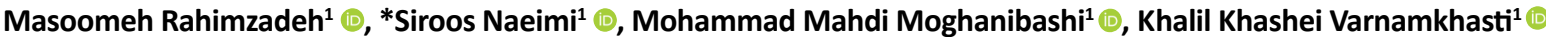

1. Department of Genetics, Colleague of Science, Kazerun Branch, Islamic Azad University, Kazerun, Iran.

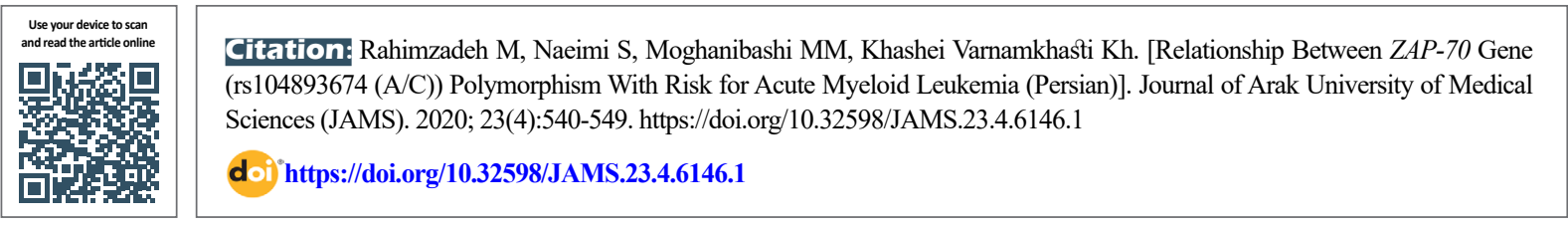

\section{(i) $\$$}

Article Info:

Received: 11 Apr 2020

Accepted: 17 May 2020

Available Online: 01 Oct 2020

Keywords:

\section{Acute myeloid}

leukemia, ZAP-70,

Leukemia, Polymor-

phism

\section{A B STRACT}

Background and Aim In acute myeloid leukemia, a large number of immature cells develop, which can related to some single nucleotide polymorphisms presence in positions of genes that encodes enzymes involved in cell activation and evolution signaling pathways. In this study, the association of rs 104893674 $(A / C)$ polymorphism with the risk of Acute Myeloid Leukemia (AML) in samples obtained from Fars and Isfahan Province hospitals was investigated.

Methods \& Materials In the present case-control study conducted at Islamic Azad University of Kazerun in 2019, 94 AML patients and 99 age and sex-matched healthy individuals were enrolled. The rs104893674 (A / C) polymorphism was determined by Tetra Primer ARMS PCR method. Data were analyzed by SPSS (version23) software using Chi-square statistical test.

Ethical Considerations This study with research ethics code IR.IAU.KAU.REC.1398.051 has been approved by Research Ethics Committee of Islamic Azad University of Kazerun.

Results The results of this study showed a significant, allele and genotype-specific Association between rs104893674 (A / C) polymorphism with risk of AML. Thus, there are more likely to develop AML in AC genotype, individuals with $A$ allele at this polymorphic site $(P=0.000)$.

Conclusion The association of acute myeloid leukemia with the genetic polymorphism of the ZAP-70 protein can be considered as an option for prognosis of this complication in susceptible individuals.

\section{Extended Abstract}

\section{Introduction}

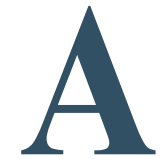

cute myeloid leukemia, with an annual incidence of about 3.5 per 100,000 people, is the most common form of acute leukemia in adulthood [4, 5]. In this heterogeneous cancer, in terms of phenotype and genotype, due to genetic changes in blood precursor cells, the natural growth and differentiation of these cells is altered and causes the accumulation of abnormal and immature myeloid cells in the bone marrow and peripheral blood [6].

Polymorphisms in a gene locus expressing proteins involved in the development and activation of myeloid cells may play a role in the immaturity of these cells. The ZAP-70 gene provides instructions for the production of the zeta-chain-dependent protein kinase, which is part of the signaling pathway for the evolution and activation of blood cells. rs $104893674(\mathrm{~A} / \mathrm{C})$ is a single nucleotide polymorphism of the $Z A P-70$ gene, which due to its location inside the exon can directly increase

\section{${ }^{*}$ Corresponding Author:}

Siroos Naeimi, PhD.

Address: Department of Genetics, Colleague of Science, Kazerun Branch, Islamic Azad University, Kazerun, Iran.

Tel: +98 (917) 1391420

E-mail: naeimis@kau.ac.ir 
or decrease the function of the gene and subsequently increase or decrease the susceptibility to disease.

\section{Materials and Methods}

This case-control study was performed on 94 patients with acute myeloid leukemia as a case group and 99 healthy individuals as a control group. All adults with acute myeloid leukemia diagnosed by a specialist, were included in the patient group. Also, people without cancer who were age- and sex-matched with cancer patients were included in the control group. Individuals with a history of any internal disease were excluded from the study. A total of $5 \mathrm{ml}$ of peripheral blood was collected from the subjects. DNA was then extracted by "salting out" method and transferred to the freezer at $-20^{\circ} \mathrm{C}$. The quality of the extracted DNA was determined on $1 \%$ agarose gel and the rs104893674 (A/C) polymorphism was determined using Tetra-primer ARMS PCR technique. Data were analyzed using SPSS v. 23 and Chi-square statistical test.

\section{Results}

The results of Tetra-primer ARMS PCR test showed 169 bp amplified fragments for $\mathrm{CC}$ genotype and $237 \mathrm{bp}$ for $\mathrm{AC}$ genotype resulting from ZAP-70 gene amplification at position rs104893674 (A/C). Since no AA genotype was found

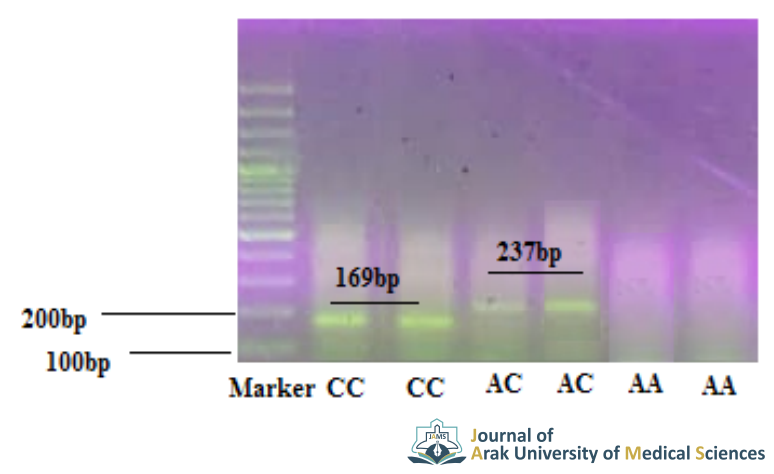

Figure 1. ARMS-PCR product from $Z A P-70$ gene amplification at rs104893674 (A/C) position

in the samples, no amplified product was shown on the gel. The results of chi-square test showed a significant relationship between the control group and the patient group in the frequency of both alleles $\mathrm{A}$ and $\mathrm{C}$ in the polymorphism rs 104893674 position $(\mathrm{P}=0.000)$. There was a significant relationship between the control group and the patient group in the frequency of $\mathrm{CC}$ and $\mathrm{AC}$ genotypes in the polymorphism rs 104893674 position $(\mathrm{P}=0.000)$ (Table $1 \& 2)$.

\section{Discussion and Conclusion}

In acute leukemia, the bone marrow produces large amounts of immature white blood cells, and the normal production of white blood cells stops, leading to a loss of

Table 1. Comparison of the frequency of alleles A and C at the rs 104893674 polymorphism position in patients

\begin{tabular}{cccccc}
\hline \multirow{2}{*}{ Allele } & \multicolumn{3}{c}{ No. (\%) } & \multicolumn{2}{c}{ The Ratio of Chances } \\
\cline { 2 - 5 } & Patient Group & Control Group & Total & (High Domain - Low Domain) & P \\
\hline A & $94(50)$ & $62(31)$ & $156(40.4)$ & $2.19(1.44-3.32)$ & 0.000 \\
C & $94(50)$ & $136(68)$ & $230(59.6)$ & $1.47(1.20-1.80)$ & 0.000 \\
Total & $188(100)$ & $198(100)$ & $386(100)$ & - & - \\
\hline
\end{tabular}

Journal of

Arak University of Medical Sciences

Table 2. Comparison of the frequency of CC, AC and AA genotypes at rs104893674 polymorphism position in patients

\begin{tabular}{cccccc}
\hline \multirow{2}{*}{ Genotype } & \multicolumn{1}{c}{ No. (\%) } & \multicolumn{2}{c}{ The Ratio of Chances } & P \\
\cline { 2 - 5 } & Patient Group & Control Group & Total & (High Domain - Low Domain) \\
\hline AC & $94(100)$ & $62(62.6)$ & $156(80.8)$ & $1.57(1.37-1.85)$ & 0.000 \\
CC & $0(0.0)$ & $37(37.4)$ & $37(37.4)$ & $1.09(0.66-0.97)$ & 0.000 \\
AA & $0(0.0)$ & $0(0.0)$ & $0(0.0)$ & - & 0.000 \\
Total & $94(100)$ & $99(100)$ & $193(100)$ & - & - \\
\hline
\end{tabular}


the body's ability to fight disease [2]. Given that research has linked leukemia to genetics, one of the most important and accurate ways to diagnose and predict the disease is to use people's DNA and genetic information [15].

Following the argument that rs104893674 mononucleotide polymorphism is located within the exon and can directly increase or decrease gene function and consequently increase or decrease susceptibility to disease, the results of the present study are significantly associated with genotypic specificity and showed an allele for rs104893674 polymorphism with a risk of acute myeloid leukemia. It was found that people with AC genotype and " $\mathrm{A}$ " allele in this polymorphic position are more prone to acute myeloid leukemia. Therefore, considering the significant association of rs104893674 polymorphism with the risk of acute myeloid leukemia, it can be concluded that genetic markers are strong predictive strategies with high sensitivity for early detection of acute myeloid leukemia (Figure 1).

\section{Ethical Considerations}

\section{Compliance with ethical guidelines}

This study was ethically approved by the ethics committee of the Vice Chancellor for Research, Islamic Azad University, Kazerun Branch (Code: IR.IAU.KAU.REC.1398.051).

\section{Funding}

The present paper was extracted from the Msc. thesis of the first author, Department of Genetics, Faculty of Science, Kazerun Branch, Islamic Azad University.

\section{Authors' contributions}

All authors equally contributed to preparing this article.

\section{Conflicts of interest}

The authors declared no conflict of interest.

\section{Acknowledgements}

The authors would like to thank the Vice Chancellor for Research of the Azad University, Kazerun Branch and all who helped with this research. 


\title{
ارتباط يلىمورفيسم رن (rs104893674"A/C") ZAP-70) با خطر ابتلا به لوسمى ميلوئيدى حاد
}

\author{
معصومه رحيمزاده' (1) "نيروس نعيمى' (1)، محمدمهلى مغنىباشى' (1)، خليل خاشعى ورنامخواستى
}

ا. كروه رُنتيك، دانشكده علوم بايايه، واحد كازرون، دانشَّاه آزاد اسلامى، كازرون، ايران.

\begin{abstract}
965)

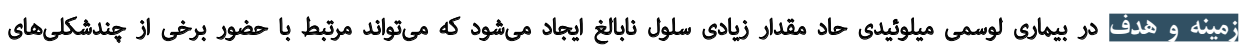

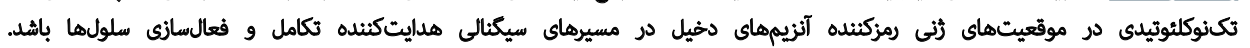

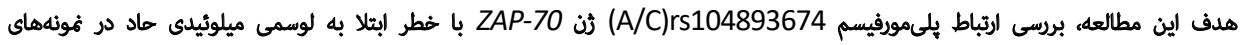

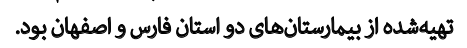

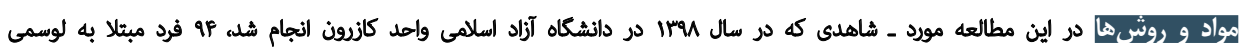

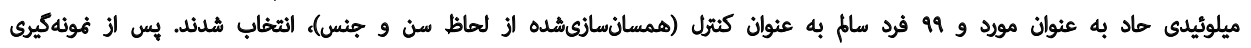

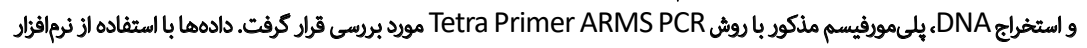

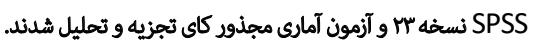

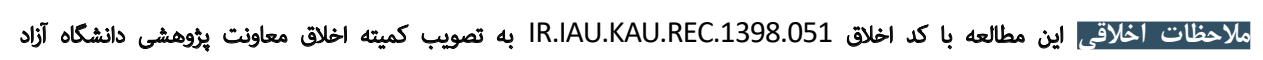
اسلامي والحد كانرين رسيد.

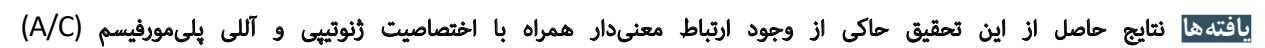

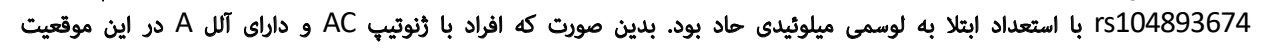

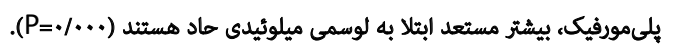

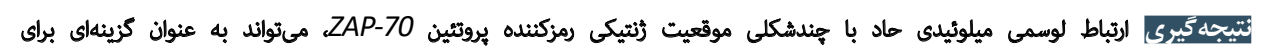
بيش آكهي اين عارضه در اقراد مستُعد مدنظر قرار كيرد.
\end{abstract}

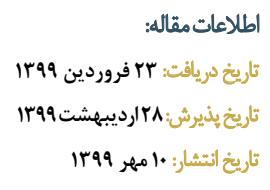
رشد و قُايز طبيعى اين سلولها دهار تغيير شده و باعث تجمع

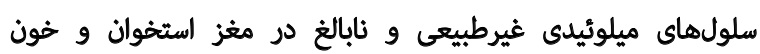

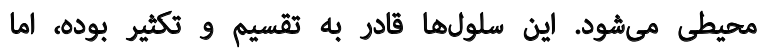

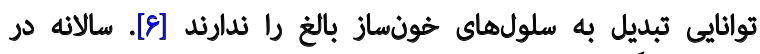

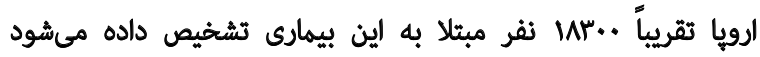

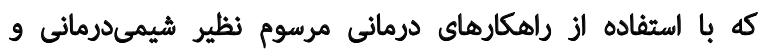

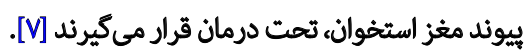

تمقيقات متعدد انجامشده در سراسر جهان و هُهند مطالعه

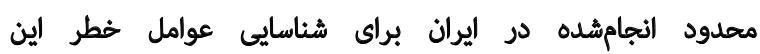

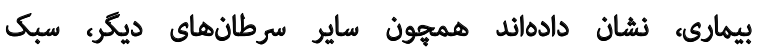

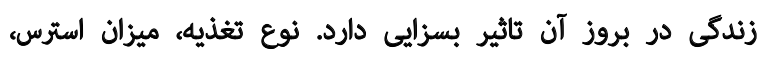

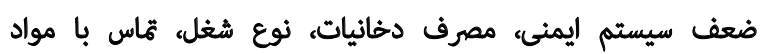

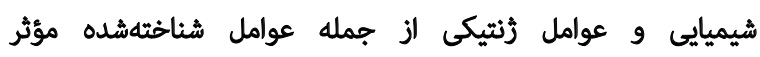

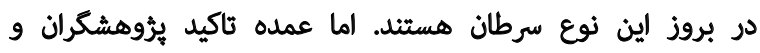

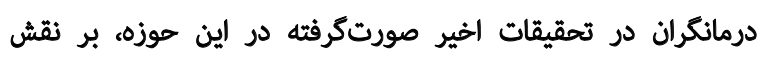

\section{كليدواروهها:

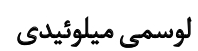

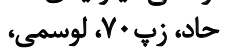 \\ يليمورفيسم حادن} . 


\section{موادو روشها}

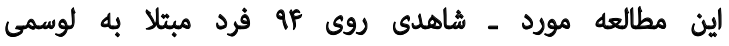

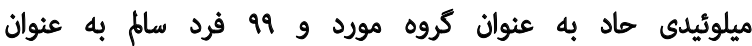

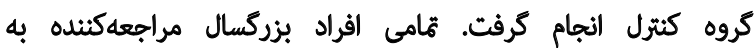

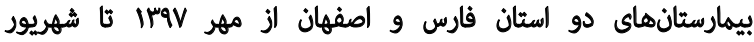

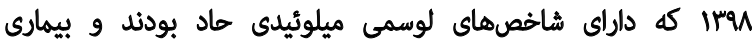

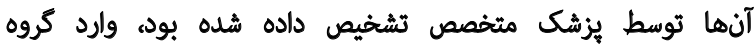

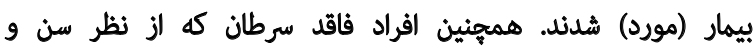

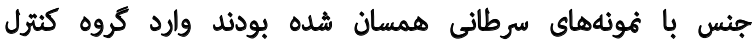

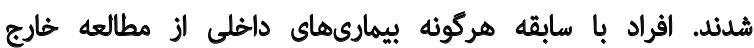

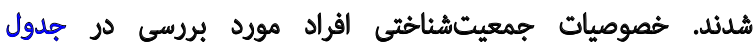
شماره ا الراثه شده استب.

يس از اخذ رضايتنامه از افراد مورد مطالعه و ير كردن يرسشنامه،

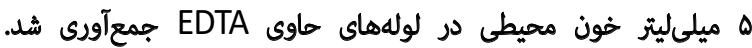

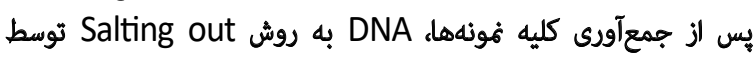
كيت شركت GeNet Bio ساخت كره جنوبى استخراج شد. سيس

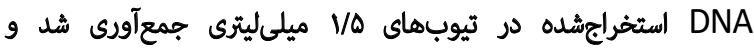

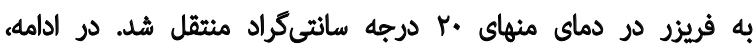

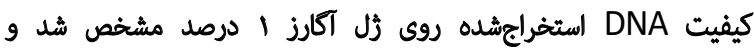

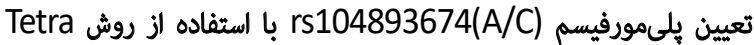
Primer ARMS PCR

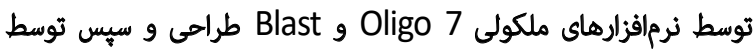
شركت Macrogen سنتز شده بود، انجام كرفت (جدول شهاره r)

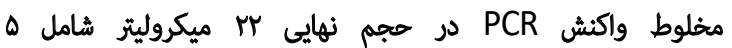

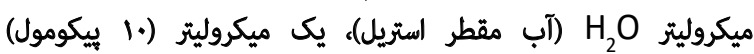

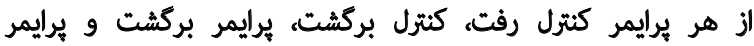

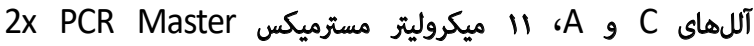
(Ampliqon, Odense, Denmark) Mix Red. Mg cl2 ميكروليتر DNA رنوميك آماده شد و تحت برنامه دماى - زمانى مربوطه قرار كرفت (جدول شهاره"r).

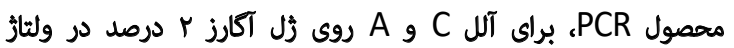

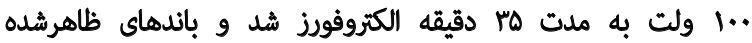
با استفاده از Klac CD Gel Documentation System مشاهده
عوامل رُنتيكى در بروز لوسمى حاد ميلوئيدى است [N]]. الز جمله

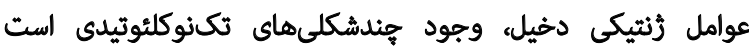

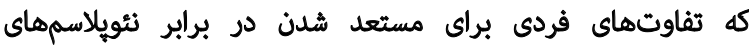

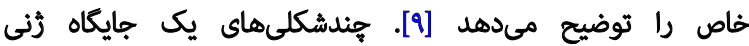

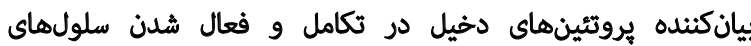

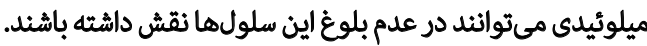

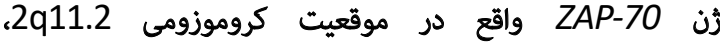

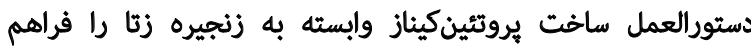

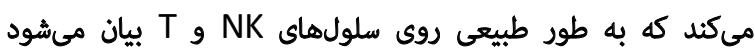

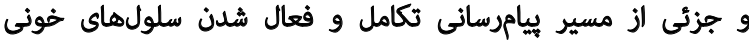

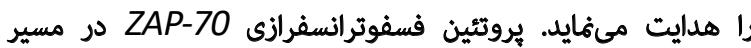

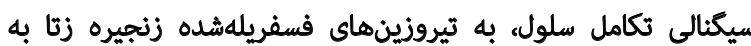

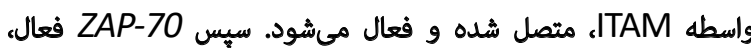

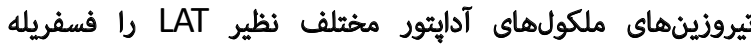

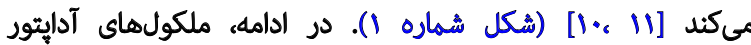

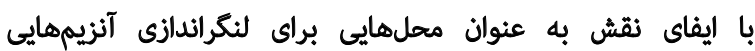

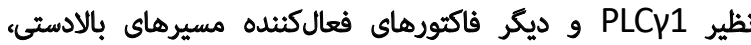

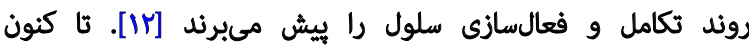

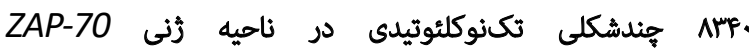
شئاسيى شده الست و مطالعات فراوانى به نقش يروتئين

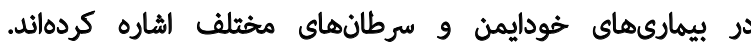

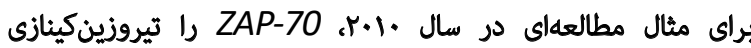

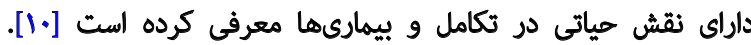

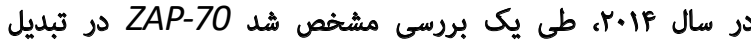

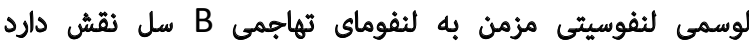

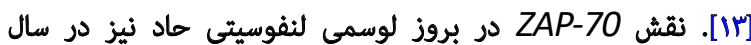

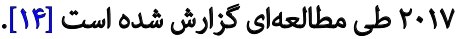

rs104893674(A/C) ZAP-70

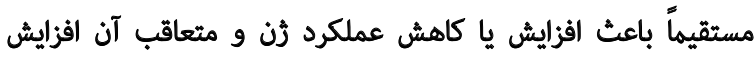

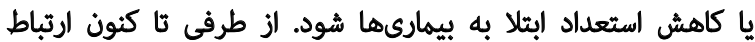

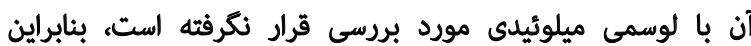

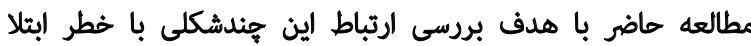

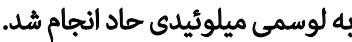

شدند (تصوير شهاره 1).

جدول ا. خصوصيات جمعيتشناختى افراد سالم و بيمار مبتلا به لوسمى ميلوئيدى حاد

\begin{tabular}{|c|c|c|}
\hline \multicolumn{2}{|c|}{ تعداد (ميانكين سنى) } & \multirow{2}{*}{ جنس } \\
\hline نمونههاى سالم (n=99) & نمونههاي بيمار (n=9) & \\
\hline \&) $(\Delta \Delta / \theta)$ & $\Delta \Lambda(\Delta) / \Lambda)$ & مرد \\
\hline re $(F T / \Delta)$ & $r e(R P / V)$ & نن \\
\hline
\end{tabular}


جدول r. مشخصات يرايمر هاي مورد مطالعه

\begin{tabular}{|c|c|c|c|c|}
\hline j) & نام آغازك & توالى أغازئ '-3' & 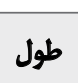 & Tlo \\
\hline \multirow{5}{*}{$\begin{array}{c}\text { ZAP-70 } \\
(\text { (rs104893674(A/C)) }\end{array}$} & F.rs104893674C & 5'- CTTCCGCAAGTTCTCCAGC-3' & 19 & $\Delta q$ \\
\hline & F.rs104893674A & 5'-CTTCCGCAAGTTCTCCAGA -3' & 19 & $\Delta V$ \\
\hline & R.rs104893674 & $5^{\prime}$ - CGATGAAGGCCATGACCTC-3' & 19 & $\Delta 9$ \\
\hline & Control-F & $5^{\prime}$-CCTCTGCACAGTTTGGAC - $3^{\prime}$ & M & $\Delta F$ \\
\hline & Control-R & $5^{\prime}$-TCTGTCCAGCAATCCAGG - $3^{\prime}$ & $M$ & $\Delta F$ \\
\hline
\end{tabular}

سلولهاى سفيد خونى معمولًا در صورت نياز بدن، به طريقى

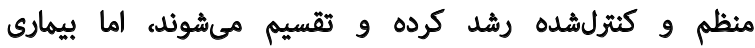

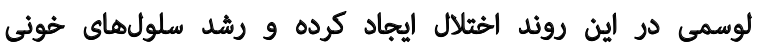

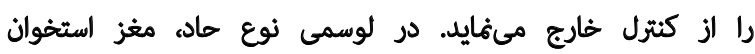

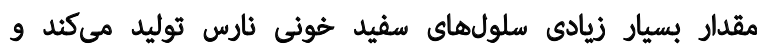

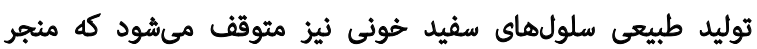

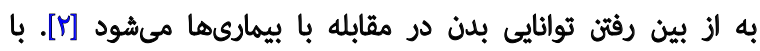

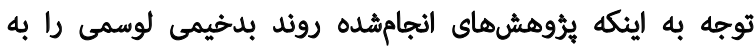

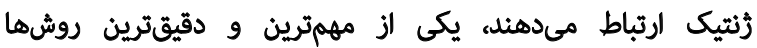

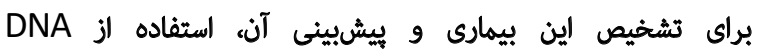

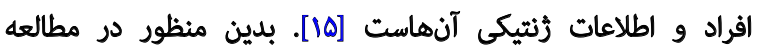

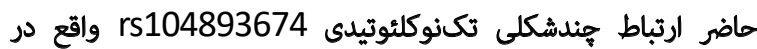

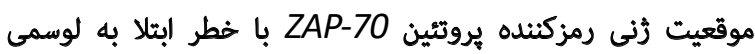

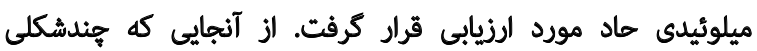

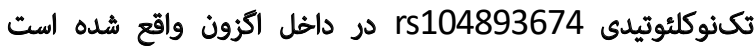

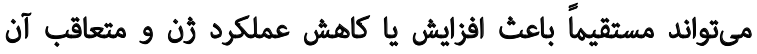

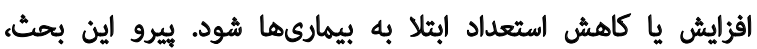

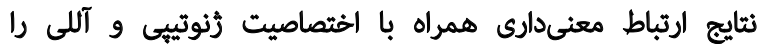
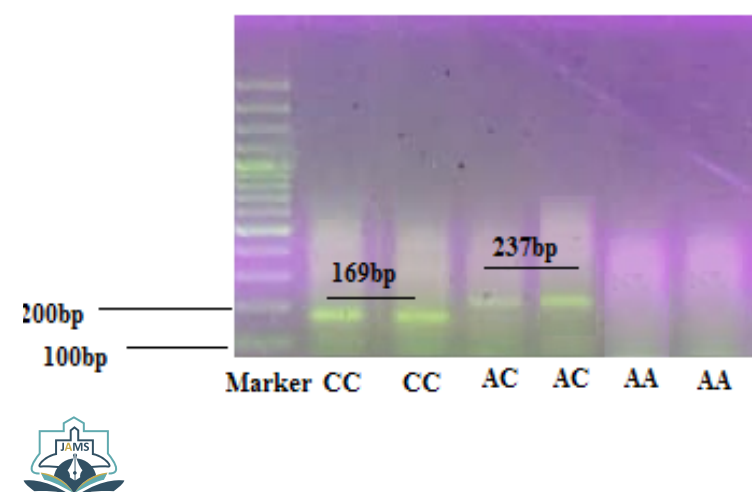

تصوير ا. محصول RCP-SMRA حاصل از تكثير دُن ZAP-70 در موقعيت rs104893674(A/C)
تجزيه و تحليل دادهها با كمك ثرم|فزار SPSS نسخه سب و آزمون آمارى مجذور كاي صورت كرفت.

Lator

\section{ARMS-PCR}

نتايج تست Tetra Primer ARMS PCR نشاندهنده قطعات تكثيريافتهاى به طول

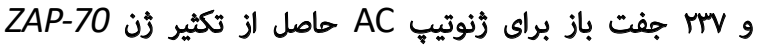

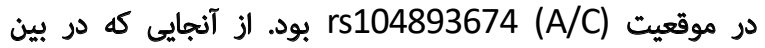
ثمونهها افرادى با رُنوتيب AA يافت نشد، محصول تكثيريافتهاي

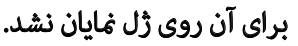

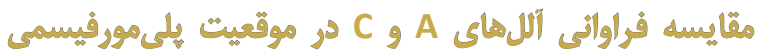

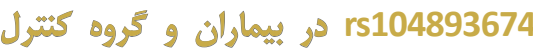

نتايج حاصل الز آزمون مجذور كاى الرتباط معنىدارى را بين

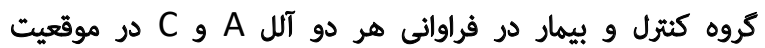

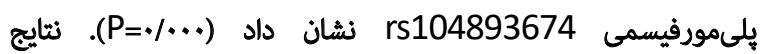

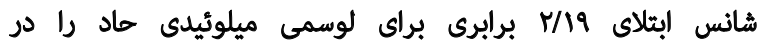

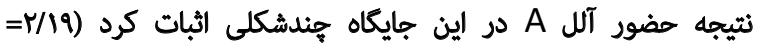

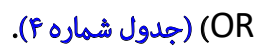

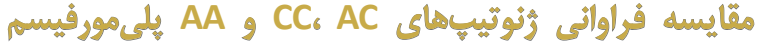

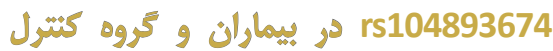

نثايج حاصل الز آزمون مجذور كاى الرثباط معنىدارى را بين

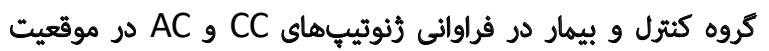

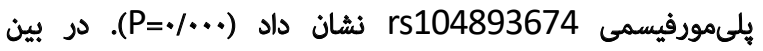

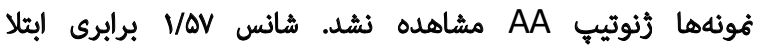

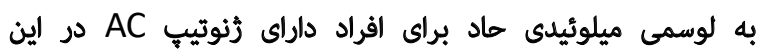

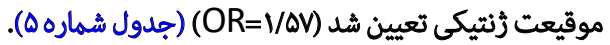


جدول r. برئامه دمايي - زمانى PCR جهت تكثير قطعه Pro4893674(A/C)

\begin{tabular}{|c|c|c|c|c|}
\hline تعداد سيكلها & زمان & دما (درجه سانتى & برثامه & رديف \\
\hline$=$ & ه دقيقه & qf & دناتوراسيون الوليه & 1 \\
\hline \multirow{3}{*}{ rr } & . بانيه & q4 & دناثوراسييون & $r$ \\
\hline & . ثانيه P. & $\Delta V / A$ & أتيلينى & $r$ \\
\hline & . بانيه & $n$ & اكستنشن & f \\
\hline 1 & ه ه ثانيه & $n$ & اكستنشن نهايى & $ه$ \\
\hline
\end{tabular}

اكزوم حاصلشده طي مطالعهاي در سال

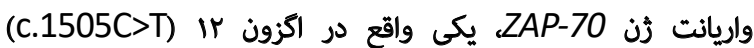

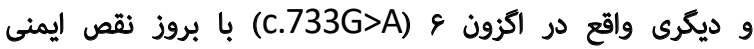

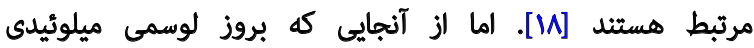

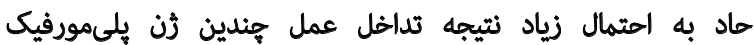

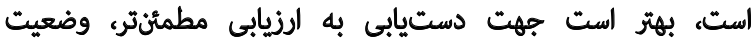

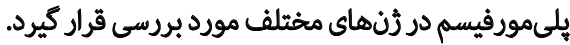

براى هندشكلى rs104893674 با خطر ابتلا به لوسمى ميلوثيدى

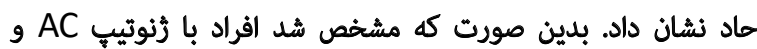

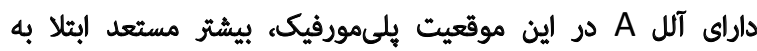

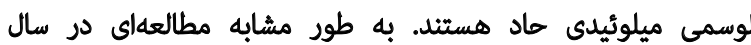

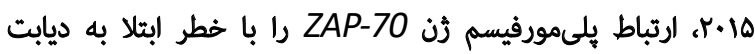

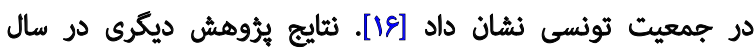

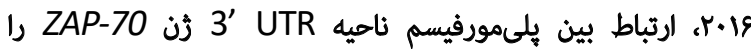

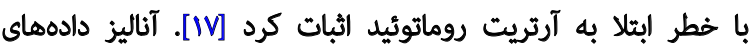

جدول P. مقايسه فراواني آللهماي A و در موقعيت يلىمورفيسم rs104893674 در بيماران مبتلا به لوسمى ميلوئيدى حاد و كروه كثترل

\begin{tabular}{|c|c|c|c|c|c|}
\hline \multirow{2}{*}{ معنى سطحى } & \multirow{2}{*}{ (دامنه بإيبن - دامنه بالا) } & \multicolumn{3}{|c|}{ تعداد (درصد) } & \multirow{2}{*}{ آلمل } \\
\hline & & جمع & مروه كتترل & كروه بيمار & \\
\hline$\%$ & $T / 19(1 / P F-r / M T)$ & $\operatorname{la} \&\left(f \cdot / f^{\infty}\right)$ & $\operatorname{gr}(m)$ & $a f(\Delta \cdot)$ & A \\
\hline \multirow[t]{2}{*}{.1.} & $\operatorname{l} / 2 r(1 / r \cdot-1 / A \cdot)$ & $r r \cdot(\Delta Q / 8)$ & $\operatorname{lrg}(8 \lambda)$ & $q f(\Delta \cdot)$ & C \\
\hline & & $M \in(1 . *)$ & $141(1 .)$. & $M(1 \cdots)$ & جمع \\
\hline
\end{tabular}

(5)

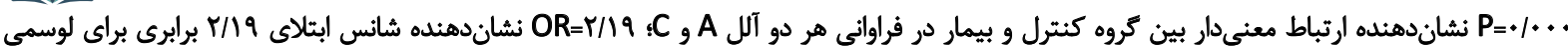

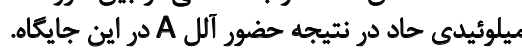

جدول ه. مقايسه فراوانى رُنوتيڤهاي AC , AC در موقعيت يلىمورفيسم rs104893674 در بيماران مبتلا به لوسمى ميلوئيدى حاد و كروه كنترل

\begin{tabular}{|c|c|c|c|c|c|}
\hline \multirow{2}{*}{ معنى سطحى } & \multirow{2}{*}{ 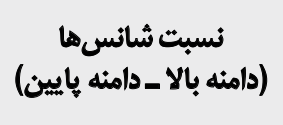 } & \multicolumn{3}{|c|}{ تعداد (درصد) } & \multirow{2}{*}{ زؤوثيب } \\
\hline & & جمع - جمع & كيروه كتترل & ئروه ييمار & \\
\hline$\%$ & $V / \Delta V(V / r V-1 / \wedge \Delta)$ & $\operatorname{lar}(\lambda+/ \Lambda)$ & $\operatorname{Er}(E T / \&)$ & $9 f(1+)$. & $A C$ \\
\hline$\%$ & $1 /+9(+/ 98-+/ 97)$ & $r V(W / F)$ & $W(r V / F)$ & $+(\cdot)$ & $\mathrm{CC}$ \\
\hline - & - & $\cdot(+)$ & $\cdot(\cdot)$ & $+(\cdot)$ & AA \\
\hline- & - & $194(1 .+1)$ & $99(1+0)$ & qf $(1+0)$ & جمع \\
\hline
\end{tabular}

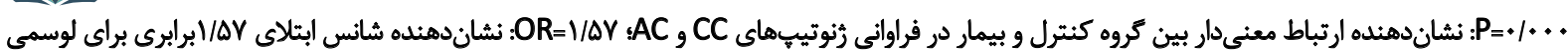

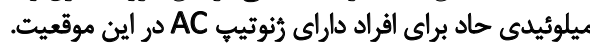




\section{نتيجليرى}

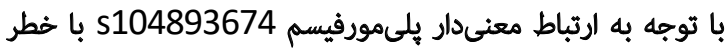

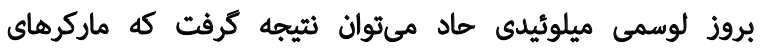

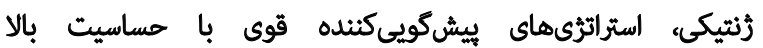
براى تشخيص زودرس لوسمى ميلوئيدى حاد هستند.

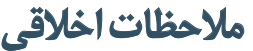

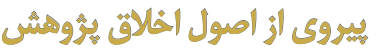
اين مطالعه با كد اخلاق تصويب كميثه اخلاق معاونت برؤوهشى دانشكاه آزاد اسلامى واحد كازرون رسيد.

$$
\text { the }
$$

اين مقاله از باياننامه كارشثاسى ارشد نويسنده اول، در كروه

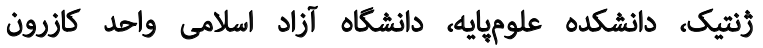
استخراج شده است.

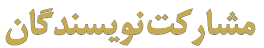

ثمامى نويسيدكان در آمادهازى اين مقاله به يك اندازه

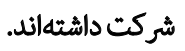

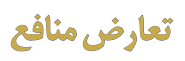

نويسندكان تصريح مىكنند هيجكونه تضاد منافعى در خصوص يزّوهش حاضر وجود ندارد.

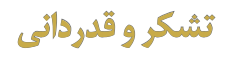

بدينوسيله از معاونت محترم يروهشى دانشكاه آزاد واحد

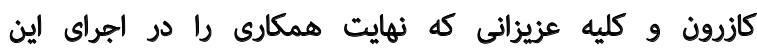
يُؤوهش داشتند، كمال تشكر و قدردانى به عمل مى آيد. 


\section{References}

[1] Babaei M, Mousavi S, Malek M, Tosi G, Zolfaghari M, Danaei N, et al. Cancer occurrence in Semnan Province, Iran: Results of a populationbased cancer registry. Asian Pac J Cancer Prev. 2005; 6(2):159-64. http://eprints.semums.ac.ir/860/1/APJCP_Volume_6_Issue_2_Pages_159-164.pdf

[2] Zand AM, Imani S, Sa'adati M, Borna H, Ziaei R, Honari H. [Effect of age, gender and blood group on blood cancer types (Persian)]. Kowsar Med J. 2010; 15(2):111-4. https://www.sid.ir/en/Journal/ViewPaper. aspx?ID=175155

[3] Robin KO, Mervin CY. Hematology, immunology and infectious disease: neonatology questions and controversies. $1^{\text {th }}$ ed. Philadelphia: Saunders; 2008. https://www.elsevier.com/books/hematology-immunology-and-infectious-disease-neonatology-questions-and-controversies/ohls/978-1-4160-3158-1

[4] Kupsa T, Horacek JM, Jebavy L. The role of cytokines in acute myeloid leukemia: A systematic review. Biomed Pap Med Fac Univ Palacky Olomouc Czech Repub. 2012; 156(4):291-301. [DOI:10.5507/ bp.2012.108] [PMID]

[5] Fauci AS, Hauser SL, Kasper DL, Longo DL, Jameson JL, Loscalzo J. Oncology and hematology. Harrison's principles of internal medicine. $19^{\text {th }}$ ed. New York: McGraw Hill Education; 2015. https://www. worldcat.org/title/harrisons-principles-of-internal-medicine/ oclc/907408102\#details-allauthors

[6] Steffen B, Muller-Tidow C, Schwable J, Berdel WE, Serve H. The molecular pathogenesis of acute myeloid leukemia. Crit Rev Oncol Hematol. 2005; 56(2):195-221. [DOI:10.1016/j.critrevonc.2004.10.012] [PMID]

[7] Parkin DM, Whelan SL, Ferlay J, Teppo L, Thomas DB. Cancer incidence in five continents. IARC Sci Publ. 2002; 8(155). https://publications. iarc.fr/Book-And-Report-Series/larc-Scientific-Publications/CancerIncidence-In-Five-Continents-Volume-VIII-2002

[8] Saffar A, Rahgozar M, Shahi F, Biglarian A. [Survival analysis of acute myeloid leukemia (Persian)]. Iran Uni Med Sci. 2015; 22(134):41-8. http:// rjms.iums.ac.ir/article-1-3921-en.html

[9] Chang FH, Tzeng DS, Lee TM, Chen TC, Hsu LS, Lung FW. Mutations in the p53 tumor suppressor gene in colorectal cancer in Taiwan. Kaohsiung J Med Sci. 2003; 19(4):151-8. [DOI:10.1016/S1607-551X(09)70464-4] [PMID]

[10] Wang H, Kadlecek TA, Au-Yeung BB, Goodfellow HES, Hsu LY, Freedman TS. ZAP-70: An essential kinase in T-cell signaling. Cold Spring Harb Perspect Biol. 2010; 2(5):a002279. [DOI:10.1101/cshperspect.a002279] [PMID] [PMCID]

[11] Smith-Garvin JE, Koretzky GA, Jordan MS. T cell activation. Annu Rev Immunol. 2009; 27:591-619. [DOI:10.1146/annurev.immunol.021908.132706] [PMID] [PMCID]

[12] Morrison DK. MAP kinase pathways. Cold Spring Harb Perspect Biol. 2012; 4(11):a011254. [DOI:10.1101/cshperspect.a011254] [PMID] [PMCID]

[13] Parikh SA, Shanafelt TD. Risk factors for Richter syndrome in chronic lymphocytic leukemia. Curr Hematol Malig Rep. 2014; 9(3):294-9. [DOI:10.1007/s11899-014-0223-4] [PMID]

[14] Alsadeq A, Fedders H, Vokuhl C, Belau NM, Zimmermann M, Wirbelauer T, et al. The role of ZAP70 kinase in acute lymphoblastic leukemia infiltration into the central nervous system. Haematologica. 2017; 102(2):346-55. [DOI:10.3324/haematol.2016.147744] [PMID] [PMCID]
[15] Torkaman A, Moghaddam Charkari N, Aghaeipour M. An approach for leukemia classification based on cooperative game theory. Anal Cell Pathol (Amst). 2011; 34(5):235-46. [DOI:10.1155/2011/212174] [PMCID]

[16] Ferjeni Z, Bouzid D, Fourati H, Stayoussef M, Abida O, Kammoun T, et al. Association of TCR/CD3, PTPN22, CD28 and ZAP70 gene polymorphisms with type 1 diabetes risk in Tunisian population: Family based association study. Immunol Lett. 2015; 163(1):1-7. [DOI:10.1016/j.imlet.2014.11.005] [PMID]

[17] Chen S-Y, Liu M-F, Wang C-R. Genetic polymorphism of 3' untranslated region of zeta-chain associated protein kinase $70 \mathrm{kDa}$ in southern Taiwanese patients with rheumatoid arthritis. Clinical rheumatology. 2016; 35(3):747-50. [DOI:10.1007/s10067-015-3044-5] [PMID]

[18] Chinn IK, Sanders RP, Stray-Pedersen A, Coban-Akdemir ZH, Kim VH$\mathrm{D}$, Dadi $\mathrm{H}$, et al. Novel combined immune deficiency and radiation sensitivity blended phenotype in an adult with biallelic variations in ZAP70 and RNF168. Front Immunol. 2017; 8:576. [DOI:10.3389/fimmu.2017.00576] [PMID] [PMCID] 
This Page Intentionally Left Blank 